\title{
Molecular survey of zoonotic Anaplasma phagocytophilum and genetic evidence of a putative novel Anaplasma species in goats from Taif, Saudi Arabia
}

\author{
Mohamed W. Ghafar ${ }^{1}$ and Sayed A. M. Amer ${ }^{2,3}$
}

1. Department of Zoonoses, Faculty of Veterinary Medicine, Cairo University, Egypt; 2. Department of Forensic Biology, College of Forensic Sciences, Naif Arab University for Security Sciences, Saudi Arabia; 3. Department of Zoology, Faculty of Science, Cairo University, Egypt.

Corresponding author: Sayed A. M. Amer, e-mail: samer@nauss.edu.sa

Co-author: MWG: mohamedghafar@hotmail.com

Received: 30-03-2019, Accepted: 06-05-2019, Published online: 03-06-2019

doi: 10.14202/IJOH.2019.54-59 How to cite this article: Ghafar MW, Amer SAM. Molecular survey of zoonotic Anaplasma phagocytophilum and genetic evidence of a putative novel Anaplasma species in goats from Taif, Saudi Arabia. Int J One Health 2019;5:54-59.

\begin{abstract}
Aim: Genus Anaplasma is of veterinary and public health importance, and its members utilize ruminants as key hosts in their epidemiology. To date, information about the occurrence and molecular identity of Anaplasma phagocytophilum and other Anaplasma species in Saudi Arabian goats is scarce. This study aimed to molecularly detect and characterize zoonotic A. phagocytophilum and other Anaplasma spp. in goats from Taif District, KSA.

Materials and Methods: Blood samples collected from 67 goats were polymerase chain reaction tested using common and A. phagocytophilum-specific primers targeting $16 \mathrm{~S}$ rRNA and msp 4 genes, respectively. Amplicons of common reactions were purified, sequenced, and analyzed.

Results: Six goats yielded positive results with common primers, whereas all animals proved negative for A. phagocytophilum. Analysis of the two successfully sequenced amplicons revealed the presence of a variant strain of Anaplasma ovis (99.52\% ID) and a new Anaplasma organism, which was clustered with Anaplasma bovis (95.9\% ID) and Aegyptianella pullorum (94.99\% ID) and distinctly separated from all other recognized species of the genus Anaplasma.

Conclusion: The tested goats proved negative for A. phagocytophilum; however, we could not confirm that the area is pathogen free. A variant strain of $A$. ovis and a putative novel Anaplasma spp. were reported raising the concern of veterinary and zoonotic potential. Other genes should be sequenced and analyzed for complete identification of the detected organisms.
\end{abstract}

Keywords: Anaplasma ovis, Anaplasma phagocytophilum, goats, phylogeny, Saudi Arabia.

\section{Introduction}

Anaplasmataceae (Rickettsiales) encompasses five recognized genera: Anaplasma, Ehrlichia, Aegyptianella, Neorickettsia, and Wolbachia. Organisms of this family are obligatory intracellular Gram-negative bacteria of veterinary and public health importance [1,2]. Anaplasma includes seven species: Anaplasma marginale, Anaplasma centrale, Anaplasma ovis, Anaplasma platys, Anaplasma bovis, Anaplasma phagocytophilum, and Anaplasma capra [3,4]. The cellular tropism, host range, vectors, and pathogenicity of these species are variables [1].

A. marginale, A. centrale, and $A$. ovis are closely related intraerythrocytic pathogens of ruminants [5-7]. $A$. marginale is known to be highly pathogenic in cattle and can result in considerable economic losses [8,9].

Copyright: Ghafar and Amer. This article is an open access article distributed under the terms of the Creative Commons Attribution 4.0 International License (http://creativecommons.org/licenses/ by/4.0/), which permits unrestricted use, distribution, and reproduction in any medium, provided you give appropriate credit to the original author(s) and the source, provide a link to the Creative Commons license, and indicate if changes were made. The Creative Commons Public Domain Dedication waiver (http:// creativecommons.org/ publicdomain/zero/1.0/) applies to the data made available in this article, unless otherwise stated.
A. centrale is less pathogenic, and, therefore, it has been used as a live vaccine against $A$. marginale in cattle $[10,11]$. A. ovis has moderate pathogenicity for sheep and goats; however, it can cause severe disease in animals exposed to stress factors [12-15]. Interestingly, variant strains of $A$. ovis were implicated in human disease in Cyprus and Iran $[16,17]$. A. platys is known to infect platelets and causes canine cyclic thrombocytopenia in dogs $[18,19]$; moreover, new closely related strains have been detected in camels, cattle, sheep, and goats, postulating that ruminants are a likely alternative host for $A$. platys [4,20-24]. Genomic evidence of $A$. platys was also identified in human patients from Venezuela, suggesting a potential public health risk [25]. A. bovis, a monocytotropic species, has been commonly recorded in cattle and buffalo from different countries [26-28]; noteworthy, $16 \mathrm{~S}$ rRNA gene sequences of $A$. bovis have been identified in Chinese goats [24]. A. phagocytophilum is a zoonotic pathogen which replicates in granulocytes of many host species, including domestic ruminants, deer, horse, dog, rodents, and humans. The pathogen causes human, canine, and equine granulocytic anaplasmosis and tick-borne fever in ruminants [29-36]. A. capra, a newly recorded novel species, was identified in goats, sheep, ticks, and humans in China; 
however, its vectors and infected cell types are unclear $[3,4,37]$. Other several candidates and unclassified Anaplasmataceae species were recently molecularly described [38-44].

According to what was stated above, it is obvious that ruminants (including goats) represent key hosts in the epidemiology of Anaplasma including zoonotic species. To date, information about the occurrence and molecular identity of Anaplasma species in Saudi Arabian domestic ruminants is scarce [45]. To the best of our knowledge, there is only one molecular survey of $A$. ovis and A. phagocytophilum in goats from Al Madinah region [46].

This study aimed to molecularly detect and characterize zoonotic A. phagocytophilum and other Anaplasma spp. in goats from Taif District, KSA.

\section{Materials and Methods}

\section{Ethical approval}

Blood samples were collected while slaughtering the goats at Taif abattoir; therefore, no ethical permission was needed.

\section{Blood samples and DNA extraction}

Blood samples were collected from 67 goats while slaughtering at Taif abattoir. These animals were residing at Taif district (approximately $21^{\circ} 26^{\prime}$ $14 " \mathrm{~N}$ and $40^{\circ} 30^{\prime} 45^{\prime \prime} \mathrm{E}$ ), KSA. The samples were sent under refrigeration to Biotechnology Laboratory at Taif University and stored at $-20^{\circ} \mathrm{C}$ until DNA extraction. According to the manufacturer's protocol, purification of DNA was executed using AxyPrep Blood Genomic DNA Miniprep Kit (Cat. No. AP-MN-BL-GDNA-250).

\section{Polymerase chain reaction (PCR) and sequencing}

All samples were examined using PCR technique with common primer pair: ECC (5'-AGA ACG AAC GCT GGC GGC AAG CC-3') and ECB (5'-CGT ATT ACC GCG GCT GCT GGC A-3'). These oligonucleotides were used to amplify the target sequence of 16S rRNA gene of Anaplasma spp. [47,48]. Animals were also tested using MAP4AP5 (5',-ATG AAT TAC AGA GAA TTG CTT GTA GG-3') and MSP4AP3 (5'- TTA ATT GAA AGC AAA TCT TGC TCC TAT G-3') primers which target $m s p 4$ gene of A. phagocytophilum [49]. PCR reactions were implemented in 25- $\mu 1$ mixtures containing $12.5 \mu \mathrm{l}$ GoTaq Green Master Mix (Promega Corporation, Madison, WI 53711-5399, USA), and 20 pmoles each primer. The thermocycle profile used in common reactions included 2-min denaturation at $94^{\circ} \mathrm{C}, 40$ cycles $(1$-min denaturation at $94^{\circ} \mathrm{C}, 2$-min annealing at $55^{\circ} \mathrm{C}$, and 30 -s extension at $72^{\circ} \mathrm{C}$ ), and additional step of 5-min final extension at $72^{\circ} \mathrm{C}$. The cycling program for the specific PCR using MAP4AP5 and MSP4AP3 primers implemented the following profile: initial 30-s denaturation at $94^{\circ} \mathrm{C}, 35$ cycles (each consisting of $30-\mathrm{s}$ denaturation at $94^{\circ} \mathrm{C}$ and combined 1-min annealing and extension at $55^{\circ} \mathrm{C}$ ), and 5 -min final extension at $72{ }^{\circ} \mathrm{C}$. Positive control samples obtained from a previous study using ECC and ECB primers [45] and negative "NO DNA" controls were included in each run. Amplicons were analyzed by agarose gel electrophoresis. Amplicons of $\sim 500 \mathrm{bp}$ and $849 \mathrm{bp}$ indicate positive results for common and specific reactions, respectively.

\section{Sequencing and analysis}

According to the manufacturer's instructions, target amplicons of positive common primer samples were extracted from agarose gel using FavorPrep Gel Purification Mini Kit (Cat. No. FAGPK001). Purified products were subjected to bidirectional sequencing using Macrogen facilities.

\section{Sequence analysis}

BLAST search was performed (http://blast. ncbi.nlm.nih.gov/Blast.cgi) to investigate homologies with sequences available in database. The obtained DNA sequences were aligned using MAFFT [50]. The unalignable and gap-containing sites were deleted so that $300 \mathrm{bp}$ were left for the analysis. The neighbor-joining phylogenetic tree was constructed with bootstrap analysis of 1000 replicates to represent the evolutionary history of the taxa analyzed [51]. The accession numbers used for comparison with our detected strains are shown in the phylogenetic tree.

\section{Nucleotide sequence accession numbers}

The partial 16S rRNA nucleotide sequences obtained in the current study were registered at GenBank under the following accession numbers: LC467272 (Anaplasma spp. MWG-2019, Ghafar-G24 strain) and LC467273 (Anaplasma spp. MWG-2019, Ghafar-G25 strain).

\section{Results}

Of 67 goats, 6 (9\%) yielded positive results when PCR tested using common primers, whereas all of animals proved negative for A. phagocytophilum. Two positive common reaction amplicons were successfully sequenced, and BLAST search of their partial 16S rRNA gene sequences showed that there were no $100 \%$ identical sequences; therefore, the new names "Anaplasma spp. MWG2019, Ghafar-G24" and Anaplasma spp. MWG-2019, Ghafar-G25" were assigned. Ghafar-G24 possessed highest similarity (100\% QC, 0.0 E-value, $99.52 \%$ ID) with A. ovis strain (JQ917900) detected in ticks from China. However, Ghafar-G25 showed highest identity (100\% QC, 0.0 E-value, 96.13\% ID) with A. bovis strain (KP314239) detected in Chinese ticks and with uncultured Anaplasma spp. (LC066137) detected in ticks from Bangladesh. Similarity features of our detected strains with species used in the phylogenetic tree are presented in Table-1. Phylogenetic analysis with recognized species representing Anaplasmataceae (Figure-1) revealed that Ghafar-G24 strain is closely related to and clustered 
Table-1: Similarity features of the detected Anaplasma organisms to recognized species used in the phylogenetic tree. (Accessed March 16, 2019).

\begin{tabular}{|c|c|c|c|c|c|c|c|c|}
\hline \multirow[t]{2}{*}{ Accession } & \multirow[t]{2}{*}{ Organism } & \multirow[t]{2}{*}{ Isolate/strain } & \multicolumn{3}{|c|}{$\begin{array}{c}\text { Anaplasma spp. } \\
\text { Ghafar-G24 }\end{array}$} & \multicolumn{3}{|c|}{$\begin{array}{c}\text { Anaplasma spp. } \\
\text { Ghafar-G25 }\end{array}$} \\
\hline & & & QC (\%) & $\mathbf{E}$ & ID (\%) & QC (\%) & $\mathbf{E}$ & ID (\%) \\
\hline JQ917900 & Anaplasma ovis & WYG59 & 100 & 0.0 & 99.52 & 100 & 0.0 & 94.53 \\
\hline AY262124 & Anaplasma ovis & - & 100 & 0.0 & 99.28 & 100 & 0.0 & 94.53 \\
\hline JF514507 & Anaplasma ovis & $54 y-S V 161$ & 73 & $3 e-164$ & 99.67 & 72 & $7 e-156$ & 96.86 \\
\hline KU686784 & Anaplasma centrale & Uganda KT5 & 100 & 0.0 & 98.81 & 99 & 0.0 & 94.76 \\
\hline AF414869 & Anaplasma centrale & South Africa & 100 & 0.0 & 98.81 & 100 & 0.0 & 94.76 \\
\hline AB916498 & Anaplasma marginale & Ghafar-1Catl-KSA & 100 & 0.0 & 98.33 & 99 & 0.0 & 94.95 \\
\hline AB916499 & Anaplasma marginale & Ghafar-6Catl-KSA & 100 & 0.0 & 97.85 & 100 & 0.0 & 95.01 \\
\hline KM206273 & Anaplasma capra & HLJ-14 & 100 & 0.0 & 95.71 & 100 & 0.0 & 93.64 \\
\hline MH762077 & Anaplasma capra & AK-Rm-429 & 100 & 0.0 & 95.71 & 100 & 0.0 & 93.64 \\
\hline JN558824 & Anaplasma bovis & G49 & 100 & 0.0 & 94.03 & 100 & 0.0 & 95.90 \\
\hline KP314251 & Anaplasma bovis & tick 18/China/2013 & 100 & 0.0 & 94.27 & 100 & 0.0 & 95.90 \\
\hline U02521 & Anaplasma phagocytophilum & Webster & 100 & 0.0 & 93.82 & 100 & 0.0 & 95.01 \\
\hline KC800985 & Anaplasma phagocytophilum & $9 B 13$ & 100 & 0.0 & 94.30 & 99 & 0.0 & 95.45 \\
\hline AY125087 & Aegyptianella pullorum & - & 100 & 0.0 & 93.79 & 100 & 0.0 & 94.99 \\
\hline AY077619 & Anaplasma platys & Okinawa & 100 & 0.0 & 93.81 & 99 & 0.0 & 95.44 \\
\hline MF289478 & Anaplasma platys & YY36 & 100 & 0.0 & 93.57 & 100 & 0.0 & 95.67 \\
\hline KF843825 & Candidatus Anaplasma camelii & Camel_7 & 100 & 0.0 & 93.57 & 100 & 0.0 & 95.69 \\
\hline KF843823 & Candidatus Anaplasma camelii & Camel_2 & 100 & 0.0 & 93.57 & 100 & 0.0 & 95.69 \\
\hline AB196302 & Ehrlichia muris & FN2619 & 100 & $8 e-162$ & 90.61 & 100 & $2 e-173$ & 91.05 \\
\hline U96436 & Ehrlichia ewingii & 95E9-TS & 87 & $8 e-162$ & 94.02 & 100 & $2 e-173$ & 91.05 \\
\hline EU826516 & Ehrlichia chaffeensis & clone 16S_Echaf_Ap & 87 & $2 e-163$ & 94.29 & 100 & $6 e-173$ & 91.01 \\
\hline U03777 & Ehrlichia ruminantium & Ball3 --1 & 100 & $1 e-155$ & 89.83 & 100 & $4 e-175$ & 91.42 \\
\hline M73221 & Ehrlichia canis & - & 87 & $4 e-160$ & 93.75 & 100 & $5 e-179$ & 91.89 \\
\hline EU810404 & Candidatus Neoehrlichia mikurensis & - & 100 & $6 e-163$ & 90.93 & 100 & $1 e-179$ & 92.05 \\
\hline AF179630 & Wolbachia pipientis & - & 99 & $4 e-135$ & 87.08 & 100 & $7 e-158$ & 89.12 \\
\hline U12457 & Neorickettsia helminthoeca & - & 100 & $3 e-121$ & 84.96 & 100 & $3 e-136$ & 86.10 \\
\hline L36105 & Rickettsia conorii & Moroccan & 100 & $9 e-117$ & 84.49 & 100 & $9 e-122$ & 84.28 \\
\hline
\end{tabular}

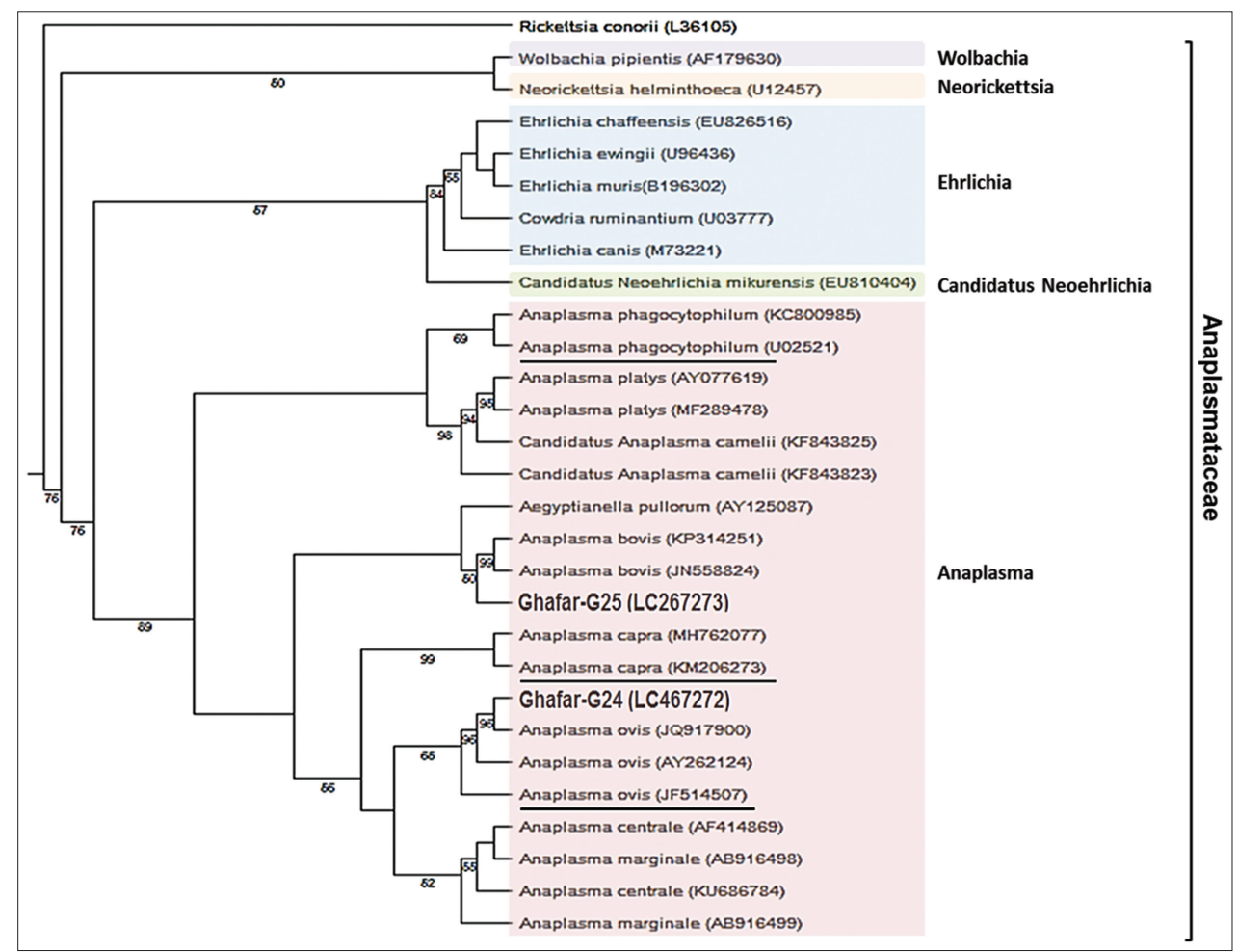

Figure-1: Neighbor-joining phylogenetic analysis of the 16S rRNA gene of detected Anaplasma spp. strains (bold) with selected designated Anaplasmataceae spp. Numbers at the nodes refer to bootstrap probabilities when they are above $50 \%$. GenBank accession numbers are shown in parentheses and human pathogens are underlined.

with A. ovis of both animal and human origin. The phylogeny also placed Ghafar-G25 strain on a distinct, separate branch within a clade containing A. bovis and Aegyptianella pullorum. 


\section{Discussion}

To date, very little is known about the magnitude of Anaplasma pathogens in Saudi Arabia. Few studies concerned microscopic examination of blood smears detected these bacteria in camel $(40.5 \%)$, cattle $(0.98 \%, 1 \%$, and $3.4 \%)$, and sheep (2\%) [52-54]. Other two serological surveys demonstrated the occurrence of $A$. marginale in camel (8.57\% and 14\%) [55], as well as $A$. ovis and $A$. phagocytophilum in sheep and goats [46]. In addition, only three molecular studies were performed to elucidate the molecular identity of Anaplasma spp. in the Kingdom [20,45,46]. Noteworthy, to the best of our knowledge, there is no study of any kind was performed on anaplasmosis in the human population. Therefore, the role of Anaplasma spp. in both animal and human medicine in KSA is not clear. In the present study, we tried to molecularly identify zoonotic $A$. phagocytophilum and other occurring Anaplasma spp. in goats residing in Taif district, KSA.

16S rRNA gene-based PCR and sequencing were employed in our experiment. This molecular technique proved invaluable in the detection and taxonomic classification of newly discovered bacteria and organisms that are difficult to grow in the laboratory. This is attributed to the fact that $16 \mathrm{~S}$ rRNA gene is less variable and therefore is sensitive to phylogenetically discriminate between different species $[1,56]$.

The negative detection of $A$. phagocytophilum in this study is consistent with the previous study conducted in the area to detect this pathogen in camel, cattle, and sheep [45]. Several plausible explanations could account for this negative result. The first, most likely, explanation is that Taif district is free of the disease due to the absence of competent vector in the area. The second, least likely, explanation is that the pathogen is present in low prevalence rate, but using of relatively small-sized sample (67 goats) led to the production of a biased result. The third, unlikely, explanation is that blood samples were collected after a short duration of bacteremia, and therefore, detection of the organism was impossible.

Six goats yielded positive results in PCR using common primers. BLAST search and phylogeny of the two successful sequences (Ghafar-G24 and Ghafar-G25) showed that the detected organisms belong to Anaplasma but distinct from all established species.

Ghafar-G24 clustered with $A$. ovis strains of tick, sheep, and human origin with identity ranged from $99.28 \%$ to $99.67 \%$ (Figure-1 and Table-1), suggesting that this organism is a variant strain of $A$. ovis. The variation in the short sequenced fragment (300 bp) may have a great impact on ecology and pathogenicity of the present strain, especially when associated with other genetic differences in protein-coding genes. Unfortunately, the clinical history of the tested goats was unavailable. Given the previous information, we cannot confirm that Ghafar-G24 strain can cause animal or human disease; however, the veterinary and human public health impact should be considered.

Ghafar-G25 strain showed genetic distance from other known Anaplasma species with highest relatedness (96.13\% identity) to A. bovis and uncultured Anaplasma spp. Phylogeny clustered Ghafar-G25 strain with strains of $A$. bovis and $A$. pullorum (Figure-1). Noteworthy, A. pullorum is still needed to be clarified whether it belongs to Anaplasma or remains in a distinct genus under Anaplasmatacea [2]. According to its level of $16 \mathrm{~S}$ rRNA gene divergence and the cutoff value $(99.0 \%)$ for species delineation [57], this strain can be potentially classified as novel species as sequence identities varied from 93.64\% to $95.9 \%$ (Table-1) when blasted with all officially recognized Anaplasma species. Interestingly, the divergence seen in 16S rRNA gene between this Saudi Arabian strain and all known Anaplasma species is greater than the divergence seen between the established genera of Anaplasmataceae, providing strong evidence for the recognition of a putative new taxon at the genus level [41]. Given all the previous information, we cannot confirm that Ghafar-G25 constitutes a new genus or even a novel species as the formal description requires analysis of multiple other genes. Unfortunately, the amount of DNA available was limited and did not allow additional sequencing.

Detection of the novel Anaplasma agent in goats does not confirm that this animal species is a competent reservoir for this pathogen; however, this study is a crucial initial step in reservoir competence studies. Molecular detection and phylogeny of new Anaplasma species from different hosts and geographic areas are still needed for elucidating the taxonomic and phylogenetic relationships among Anaplasmataceae species. We cannot confirm that Ghafar-G25 bacteria can cause disease in animals or human; however, the veterinary health and zoonotic potential of this strain should be considered.

\section{Conclusion}

This study reports for the first time the presence of a potentially zoonotic variant strain of $A$. ovis and a putative novel Anaplasma spp. in goats from Saudi Arabia. Other multiple genes should be sequenced and analyzed to reach the formal description of the detected organisms. Other investigations are also required to elucidate the epidemiology of the newly discovered agent including competent vector and reservoir, as well as geographic distribution. Pathogenicity to animals and zoonotic importance of the organism should also be determined.

\section{Authors' Contributions}

MWG designed the study, collected the samples and materials, and performed the experiments. SAMA and MWG conducted molecular and phylogenetic analyses. Both authors wrote the manuscript. All authors read and approved the final manuscript. 


\section{Acknowledgments}

The authors are thankful to the Biotechnology Department, Taif University, for providing facilities. The authors did not receive any external fund for this study. We would like to thank the veterinarian in charge and workers at Taif abattoir for their valuable help during the collection of blood samples.

\section{Competing Interests} interests.

The authors declare that they have no competing

\section{Publisher's Note}

Veterinary World remains neutral with regard to jurisdictional claims in published institutional affiliation.

\section{References}

1. Dumler JS, Barbet AF, Bekker CP, Dasch GA, Palmer GH, Ray SC, et al. Reorganization of genera in the families Rickettsiaceae and Anaplasmataceae in the order Rickettsiales: Unification of some species of Ehrlichia with Anaplasma, Cowdria with Ehrlichia and Ehrlichia with Neorickettsia, descriptions of six new species combinations and designation of Ehrlichia equi and "HGE agent" as subjective synonyms of Ehrlichia phagocytophila. Int J Syst Evol Microbiol 2001;51:2145-65.

2. Rikihisa Y, Zhang C, Christensen BM. Molecular characterization of Aegyptianella pullorum (Rickettsiales, Anaplasmataceae). J Clin Microbiol 2003;41:5294-7.

3. Li H, Zheng YC, Ma L, Jia N, Jiang BG, Jiang RR, et al. Human infection with a novel tick-borne Anaplasma species in China: A surveillance study. Lancet Infect Dis 2015;15:663-70.

4. Yang J, Li Y, Liu Z, Liu J, Niu Q, Ren Q, et al. Molecular detection and characterization of Anaplasma spp. In sheep and cattle from Xinjiang, northwest China. Parasit Vectors $2015 ; 8: 108$.

5. Inokuma H, Terada Y, Kamio T, RaoultD, Brouqui P. Analysis of the 16S rRNA gene sequence of Anaplasma centrale and its phylogenetic relatedness to other Ehrlichiae. Clin Diagn Lab Immunol 2001;8:241-4.

6. Byaruhanga C, Collins NE, Knobel DL, Khumalo ZTH, Chaisi ME, Oosthuizen MC, et al. Molecular detection and phylogenetic analysis of Anaplasma marginale and Anaplasma centrale amongst transhumant cattle in NorthEastern Uganda. Ticks Tick Borne Dis 2018;9:580-8.

7. Renneker S, Abdo J, Salih DE, Karagenç T, Bilgiç H, Torina A, et al. Can Anaplasma ovis in small ruminants be neglected any longer? Transbound Emerg Dis 2013;60 Suppl 2:105-12.

8. Kocan KM, de la Fuente J, Blouin EF, Coetzee JF, Ewing SA. The natural history of Anaplasma marginale. Vet Parasitol 2010;167:95-107.

9. Adjou Moumouni PF, Aboge GO, Terkawi MA, Masatani T, Cao S, Kamyingkird K, et al. Molecular detection and characterization of Babesia bovis, Babesia bigemina, Theileria species and Anaplasma marginale isolated from cattle in Kenya. Parasit Vectors 2015;8:496.

10. Battilani M, De Arcangeli S, Balboni A, Dondi F. Genetic diversity and molecular epidemiology of Anaplasma. Infect Genet Evol 2017;49:195-211.

11. Rjeibi MR, Ayadi O, Rekik M, Gharbi M. Molecular survey and genetic characterization of Anaplasma centrale, A. marginale and A. bovis in cattle from Algeria. Transbound Emerg Dis 2018;65:456-64.

12. Kuttler KL. Anaplasma infections in wild and domestic ruminants: A review. J Wildl Dis 1984;20:12-20.
13. Friedhoff KT. Tick-borne diseases of sheep and goats caused by Babesia, Theileria or Anaplasma spp. Parasitologia 1997;39:99-109.

14. Tibbitts T, Goff W, Foreyt W, Stiller D. Susceptibility of two rocky mountain bighorn sheep to experimental infection with Anaplasma ovis. J Wildl Dis 1992;28:125-9.

15. Lee SH, Mossaad E, Ibrahim AM, Ismail AA, Moumouni PF, Liu M, et al. Detection and molecular characterization of tick-borne pathogens infecting sheep and goats in Blue Nile and West Kordofan states in Sudan. Ticks Tick Borne Dis 2018;9:598-604.

16. Chochlakis D, Ioannou I, Tselentis Y, Psaroulaki A. Human anaplasmosis and Anaplasma ovis variant. Emerg Infect Dis 2010;16:1031-2.

17. Hosseini-Vasoukolaei N, Oshaghi MA, Shayan P, Vatandoost H, Babamahmoudi F, Yaghoobi-Ershadi MR, et al. Anaplasma infection in ticks, livestock and human in Ghaemshahr, Mazandaran Province, Iran. J Arthropod Borne Dis 2014;8:204-11.

18. Said MB, Belkahia H, Messadi L. Anaplasma spp. In North Africa: A review on molecular epidemiology, associated risk factors and genetic characteristics. Ticks Tick Borne Dis 2018;9:543-55.

19. Vieira FT, Acosta IC, Martins TF, Filho JM, Krawczak FD, Barbieri AR, et al. Tick-borne infections in dogs and horses in the state of Espírito Santo, Southeast Brazil. Vet Parasitol 2018;249:43-8

20. Bastos AD, Mohammed OB, Bennett NC, Petevinos C, Alagaili AN. Molecular detection of novel Anaplasmataceae closely related to Anaplasma platys and Ehrlichia canis in the dromedary camel (Camelus dromedarius). Vet Microbiol 2015;179:310-4.

21. Belkahia H, Said MB, Sayahi L, Alberti A, Messadi L. Detection of novel strains genetically related to Anaplasma platys in Tunisian one-humped camels (Camelus dromedarius). J Infect Dev Ctries 2015;9:1117-25.

22. Li Y, Yang J, Chen Z, Qin G, Li Y, Li Q, et al. Anaplasma infection of Bactrian camels (Camelus bactrianus) and ticks in Xinjiang, China. Parasit Vectors 2015;8:313.

23. Zobba R, Anfossi AG, Parpaglia ML, Dore GM, Chessa B, Spezzigu A, et al. Molecular investigation and phylogeny of Anaplasma spp. In Mediterranean ruminants reveal the presence of neutrophil-tropic strains closely related to A. platys. Appl Environ Microbiol 2014;80:271-80.

24. Liu Z, Ma M, Wang Z, Wang J, Peng Y, Li Y, et al. Molecular survey and genetic identification of Anaplasma species in goats from central and Southern China. Appl Environ Microbiol 2012;78:464-70.

25. Arraga-Alvarado CM, Qurollo BA, Parra OC, Berrueta MA, Hegarty BC, Breitschwerdt EB, et al. Case report: Molecular evidence of Anaplasma platys infection in two women from Venezuela. Am J Trop Med Hyg 2014;91:1161-5.

26. Noaman V, Shayan P. Molecular detection of Anaplasma bovis in cattle from central part of Iran. Vet Res Forum 2010;1:117-22

27. Ooshiro M, Zakimi S, Matsukawa Y, Katagiri Y, Inokuma H. Detection of Anaplasma bovis and Anaplasma phagocytophilum from cattle on Yonaguni Island, Okinawa, Japan. Vet Parasitol 2008;154:360-4

28. Jilintai, Seino N, Hayakawa D, Suzuki M, Hata H, Kondo S, et al. Molecular survey for Anaplasma bovis and Anaplasma phagocytophilum infection in cattle in pastureland where sika deer appear in Hokkaido, Japan. Jpn J Infect Dis 2009;62:73-5.

29. Chen SM, Dumler JS, Bakken JS, Walker DH. Identification of a granulocytotropic Ehrlichia species as the etiologic agent of human disease. J Clin Microbiol 1994;32:589-95.

30. Jahfari S, Coipan EC, Fonville M, van Leeuwen AD, Hengeveld P, Heylen D, et al. Circulation of four Anaplasma phagocytophilum ecotypes in Europe. Parasit Vectors 2014;7:365.

31. de la Fuente J, Estrada-Peña A, Cabezas-Cruz A, Kocan KM. 
Anaplasma phagocytophilum uses common strategies for infection of ticks and vertebrate hosts. Trends Microbiol 2016;24:173-80.

32. Ochirkhuu N, Konnai S, Odbileg R, Murata S, Ohashi K. Molecular epidemiological survey and genetic characterization of Anaplasma species in Mongolian livestock. Vector Borne Zoonotic Dis 2017;17:539-49.

33. Zhan L, Cao WC, Jiang JF, Zhang XA, Wu XM, Zhang WY, et al. Anaplasma phagocytophilum in livestock and small rodents. Vet Microbiol 2010;144:405-8.

34. Dumler JS, Choi KS, Garcia-Garcia JC, Barat NS, Scorpio DG, Garyu JW, et al. Human granulocytic anaplasmosis and Anaplasma phagocytophilum. Emerg Infect Dis 2005;11:1828-34.

35. Dahmani M, Davoust B, Benterki MS, Fenollar F, Raoult D, Mediannikov O, et al. Development of a new PCR-based assay to detect Anaplasmataceae and the first report of Anaplasma phagocytophilum and Anaplasma platys in cattle from Algeria. Comp Immunol Microbiol Infect Dis 2015;39:39-45.

36. M'ghirbi Y, Bèji M, Oporto B, Khrouf F, Hurtado A, Bouattour A, et al. Anaplasma marginale and A. phagocytophilum in cattle in Tunisia. Parasit Vectors 2016;9:556.

37. Yang J, Liu Z, Niu Q, Liu J, Han R, Liu G, et al. Molecular survey and characterization of a novel Anaplasma species closely related to Anaplasma capra in ticks, Northwestern China. Parasit Vectors 2016;9:603.

38. Cicala F, Moore JD, Cáceres-Martínez J, Río-Portilla MA, Hernández-Rodríguez $\mathrm{M}$, Vásquez-Yeomans $\mathrm{R}$, et al. Multigenetic characterization of "Candidatus Xenohaliotis californiensis". Int J Syst Evol Microbiol 2017;67:42-9.

39. Dahmani M, Davoust B, Tahir D, Raoult D, Fenollar F, Mediannikov $\mathrm{O}$, et al. Molecular investigation and phylogeny of Anaplasmataceae species infecting domestic animals and ticks in Corsica, France. Parasit Vectors 2017;10:302.

40. Ehounoud CB, Yao KP, Dahmani M, Achi YL, Amanzougaghene N, Kacou N'Douba A, et al. Multiple pathogens including potential new species in tick vectors in Côte d'Ivoire. PLoS Negl Trop Dis 2016;10:e0004367.

41. Eshoo MW, Carolan HE, Massire C, Chou DM, Crowder CD, Rounds MA, et al. Survey of Ixodes pacificus ticks in California reveals a diversity of microorganisms and a novel and widespread Anaplasmataceae species. PLoS One 2015; 10:e135828.

42. Guo WP, Tian JH, Lin XD, Ni XB, Chen XP, Liao Y, et al. Extensive genetic diversity of Rickettsiales bacteria in multiple mosquito species. Sci Rep 2016;6:38770.

43. Li Y, Chen Z, Liu Z, Liu J, Yang J, Li Q, et al. Molecular survey of Anaplasma and Ehrlichia of red deer and sika deer in Gansu, China in 2013. Transbound Emerg Dis 2016;63:e228-36.
44. Qin XR, Han FJ, Luo LM, Zhao FM, Han HJ, Zhang ZT, et al. Anaplasma species detected in Haemaphysalis longicornis tick from China. Ticks Tick Borne Dis 2018;9:840-3.

45. Ghafar MW, Shobrak MY Molecular detection and characterization of Anaplasma phagocytophilum, the causative agent of human granulocytic anaplasmosis, in some animals suspected to be competent reservoirs in Taif district, Kingdom of Saudi Arabia. Life Sci J 2014;11:63-9.

46. Taha HA, Shoman SA, Alhadlag NM. Molecular and serological survey of some hemoprotozoan, rickettsial and viral diseases of small ruminants from al-Madinah al Munawarah, KSA. Trop Biomed 2015;32:511-23.

47. Kocan AA, Levesque GC, Whitworth LC, Murphy GL, Ewing SA, Barker RW, et al. Naturally occurring Ehrlichia chaffeensis infection in coyotes from Oklahoma. Emerg Infect Dis 2000;6:477-80.

48. Murphy GL, Ewing SA, Whitworth LC, Fox JC, Kocan AA. A molecular and serologic survey of Ehrlichia canis, E. chaffeensis, and E. ewingii in dogs and ticks from Oklahoma. Vet Parasitol 1998;79:325-39.

49. de la Fuente J, Massung RF, Wong SJ, Chu FK, Lutz H, Meli M, et al. Sequence analysis of the msp4 gene of Anaplasma phagocytophilum strains. J Clin Microbiol 2005;43:1309-17.

50. Katoh K, Rozewicki J, Yamada KD. MAFFT online service: Multiple sequence alignment, interactive sequence choice and visualization. Brief Bioinform 2017: 1-7.

51. Saitou N, Nei M. The neighbor-joining method: A new method for reconstructing phylogenetic trees. Mol Biol Evol 1987;4:406-25.

52. Ismael AB, Swelum AA, Khalaf AF, Alowaimer AN. First evidence of natural anaplasmosis in Camelus dromedarius in Saudi Arabia. J Camel Pract Res 2016;23:95-100.

53. El-Metenawy TM. Prevalence of blood parasites among cattle at the central area of Saudi Arabia. Vet Parasitol 2000;87:231-6.

54. Al-Khalifa MS, Hussein HS, Diab FM, Khalil GM. Blood parasites of livestock in certain regions in Saudi Arabia. Saudi J Biol Sci 2009;16:63-7.

55. Al-Gharban HA, Al-Taee HS. Seroclinical diagnosis of Anaplasma marginale bacteria in carrier Arabian onehumped camels. Bas J Vet Res 2016;15:346-59.

56. Lew AE, Gale KR, Minchin CM, Shkap V, de Waal DT. Phylogenetic analysis of the erythrocytic Anaplasma species based on 16S rDNA and groEL (HSP60) sequences of $A$. marginale, A. centrale, and $A$. ovis and the specific detection of $A$. centrale vaccine strain. Vet Microbiol 2003;92:145-60.

57. Adékambi T, Shinnick TM, Raoult D, Drancourt M. Complete rpoB gene sequencing as a suitable supplement to DNA-DNA hybridization for bacterial species and genus delineation. Int J Syst Evol Microbiol 2008;58:1807-14. 